\title{
Surface Waveguide Technology for Telecom and Biochemical Sensing
}

\author{
R.G. Heideman ${ }^{1}$, J.A. Walker ${ }^{1,2}$ \\ ${ }^{1}$ LioniX BV, P.O. Box 456, 7500 AH, Enschede, the Netherlands \\ ${ }^{2}$ JayWalker Technical Consulting, 4 Plantation Dr., Suite 110, Freehold, NJ, 07728
}

\begin{abstract}
Surface waveguides for telecom applications are typically $\mathrm{SiO}_{2}$-based, low-contrast surface waveguides because these applications are dominated by the need for low optical attenuation and low polarization effects across the 1300-1600 nm band. Conventional waveguides, however, comprise films as thick as 20 micron and have minimum bend radii of tens of millimeters. These factors make conventional waveguide circuits large and expensive, and this has limited their use to relatively few applications.

In the integrated optical sensing field, the waveguides typically used are (very) high-contrast waveguides. Here, especially $\mathrm{Si}_{3} \mathrm{~N}_{4}$-core waveguides are well-known to offer much smaller bending radii (tens to hundreds of microns) due to stronger mode confinement to the core. Since they also typically comprise sub-micron core-thickness and cladding-thickness of only a few microns, high-contrast waveguides promise lower cost than low-contrast waveguides. Their use in telecom applications has been limited, however, due to strong polarization effects. Recently, LioniX, BV has developed the TriPleX ${ }^{\mathrm{TM}}$ waveguide, which promises to be a well-suited platform for both telecom and sensing applications and is based on low-cost, CMOS-compatible LPCVD processing. TriPleX ${ }^{\mathrm{TM}}$ technology provides highcontrast waveguides with very low channel attenuation and modal birefringence that is controlled through waveguide design alone. Early experiments on typical geometries show promising waveguide characteristics (attenuation « 0.5 $\mathrm{dB} / \mathrm{cm}, \mathrm{IL} \leq-2 \mathrm{~dB}, \mathrm{PDL} \ll 1 \mathrm{~dB}$, bend radius $« 1 \mathrm{~mm})$.

In this paper, we present the characteristics of this TriPleX ${ }^{\mathrm{TM}}$ technology, and show devices that have demonstrated utility in telecom and/or sensor applications using medium and high-contrast waveguides. Experimental results for an MZI-based sensor platform, suitable for liquid or gaseous sensing, are also provided.
\end{abstract}

\section{INTRODUCTION}

Conventional integrated optic circuits typically comprise doped $\mathrm{SiO}_{2}$ for both core and cladding material. One or both of the core/cladding is doped to provide a very slight difference $(<1 \%)$ in refractive index that acts to confine propagating optical energy to the core. These low-contrast waveguides are capable of achieving very low attenuation per centimeter $(0.05 \mathrm{~dB} / \mathrm{cm}$ or better) with sufficiently low polarization effects as to make them suitable for telecommunications applications [1]. In fact, low-contrast $\mathrm{SiO}_{2}$-based waveguides have become the de-facto standard for telecom integrated optic circuits.

The widespread application of conventional surface waveguides beyond a few telecom applications has been hampered by their high-cost, however. The cost of a conventional integrated optic circuit is negatively impacted by several factors: a large real-estate requirement; challenging fabrication; and high packaging costs [2].

Since the difference between the index of refraction of the core and the cladding is very slight, a propagating optical mode is only weakly-confined to the core. Much of the propagating energy, in fact, is contained in an evanescent tail that projects into the cladding. As a result, optical circuits formed in conventional surface waveguides can not support waveguide bends tighter than 10's of millimeters in radius. In addition, the minimum waveguide spacing must be on the order of 100 microns to avoid optical cross-talk between adjacent waveguides. The real estate that a conventional optical circuit requires, therefore, is substantial.

Conventional $\mathrm{SiO}_{2}$ waveguides are quite challenging to fabricate. A typical core-layer is $8 \mu \mathrm{m}$-thick, while the upper and lower $\mathrm{SiO}_{2}$ cladding layers can exceed 20 microns in thickness. The time required to deposit such thick material layers is long, and the thickness, doping levels, and residual stress in such thick films are difficult to control. Variations of these waveguide parameters lead to signal propagation issues such as increased attenuation, polarization-dependent loss, and polarization-mode dispersion; thus, chip yield is reduced. 
Their high chip real-estate needs and high cost of production make conventional $\mathrm{SiO}_{2}$ integrated optic circuits quite expensive. Cost has dampened the enthusiasm for the deployment of integrated optic components in areas outside of telecom, as well as many within telecom. This is particularly true for components that can not be shared among multiple users.

\section{HIGH-CONTRAST WAVEGUIDE TECHNOLOGY}

Alternative materials, such as silicon oxynitride ( $\mathrm{SiON})$ and silicon nitride ( $\mathrm{SiN}$ ) might provide solutions to many of the issues with $\mathrm{SiO}_{2}$. Work at the University of Twente (Enschede, The Netherlands) and IBM's Zurich Research Lab, where the $\mathrm{SiO}_{2}$ core layer has been replaced by a layer of $\mathrm{SiON}$, demonstrates that low-loss, compact optical circuits can be made. The refractive index of SiON can be tailored from 1.46 (silicon dioxide) to 1.98 (silicon nitride) through control of the gas mixture during plasma-deposition. Therefore, SiON can be used to form waveguides with contrast that can range from $<1 \%$ to as high as $35 \%$.

Low to medium contrast ( $<1$ to $5 \%$ ) waveguides have been targeted for use in telecommunications applications. Until recently, the focus has been on developing $\mathrm{SiON}$ waveguides with a contrast of $\sim 2.5 \%$, which allows for a minimal bending radius of $\sim 500$ microns. The results have been encouraging, with low attenuation levels over the telecom bands (approaching $0.10 \mathrm{~dB} / \mathrm{cm}$ ) and low modal birefringence.

Figure 1: a) Structure of a low-contrast SiO2 core waveguide. Cover layer (top cladding) thickness is typically as much as 20 microns. b) Structure of a high-contrast SiN core waveguide. Cover layer thickness can be of order of one micron.

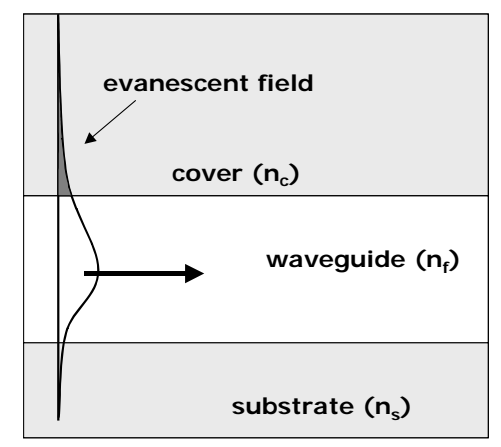

a)

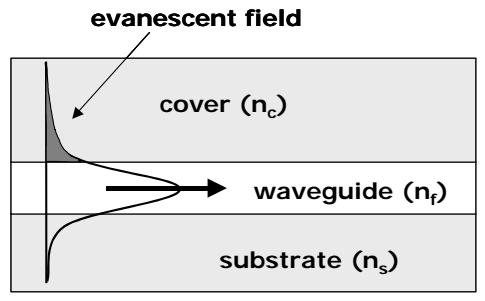

b)

For sensor applications, higher contrast (up to 35\%) waveguides are more desirable, and therefore, core compositions closer to SiN are attractive. Due to the high refractive index of SiN, a propagating optical mode is more tightly confined to the core and very tight curves can be utilized. Thin layers of SiN (of order $100 \mathrm{~nm}$ ) can be used to form single-mode channels that have very high surface sensitivity and very large modal birefringence. In addition, thinner cladding layers enable easier access to the evanescent mode which propagates in the cladding as shown in Figure 1. These factors make SiN-core waveguides attractive for optical sensing applications. Attenuation levels are roughly $0.5 \mathrm{~dB} / \mathrm{cm}$ to $1 \mathrm{~dB} / \mathrm{cm}$ in the visible, and $0.2-0.5 \mathrm{~dB} / \mathrm{cm}$ in the infrared.

Surface sensitivity of a waveguide-based optical sensor is linear with refractive index and inversely linear to waveguide thickness. Sensitivity is also inversely proportional to the wavelength of light. As a result, silicon nitride is particularly well-suited for sensing applications, as it also shows very good optical transmission at small wavelengths [3]. Unfortunately, silicon nitride is NOT well-suited to telecom applications due to its high polarization dependency.

\section{TriPleX ${ }^{\mathrm{TM}}$ TECHNOLOGY}

Recently, LioniX, BV announced a new high-contrast waveguide technology, which is suitable for both telecom and sensor applications. TriPleX ${ }^{\mathrm{TM}}$ waveguides combine the transmission characteristics of conventional $\mathrm{SiO}_{2}$ core waveguides with the cost and performance advantages of high-contrast waveguides. TriPleX ${ }^{\mathrm{TM}}$ waveguides comprise a composite core structure, which includes an inner core of $\mathrm{SiO} 2$ and an outer core of silicon nitride. This composite core behaves in a similar manner to high-contrast waveguide cores, wherein the propagating optical mode is tightly confined to the core. Through variation of design, the stress of the high-index silicon nitride can be used to control the overall stress configuration of the composite core. Simply through design choice then, the modal birefringence of the waveguide can be completely controlled over the range of $<10^{-4}$ to very high.

The cost advantages of TriPleX ${ }^{\mathrm{TM}}$ waveguides stem from the fact that they are formed via Low-Pressure Chemical Vapor Deposition (LPCVD) deposition of stoichiometric silicon nitride $\left(\mathrm{Si}_{3} \mathrm{~N}_{4}\right)$ and stoichiometric TEOS silicon 
dioxide. LPCVD-deposited stoichiometric materials are more predictable and repeatable from run-to-run than plasmadeposited materials. In addition, since LPCVD is a batch-process, rather than a single-wafer process, fabrication costs are typically much lower than for plasma-deposited films. Also, TriPleX ${ }^{\mathrm{TM}}$ waveguides comprise thin layers for core and cladding making them easier to deposit and pattern using conventional techniques. Finally, since TriPleX $\mathrm{X}^{\mathrm{TM}}$ waveguides are effectively high-contrast waveguides wherein the optical mode is tightly confined to the core, they can exhibit tight bending radii and dense waveguide layouts. Chip real-estate requirements, therefore, are reduced from similar designs in a conventional waveguide technology.

A representative TriPleX ${ }^{\mathrm{TM}}$ waveguide structure is shown in Figure 2. It comprises an LPCVD-deposited layer stack which has been patterned to form a rectangular channel waveguide structure. The waveguide essentially constitutes an "outer core" of silicon nitride $\left(\mathrm{Si}_{3} \mathrm{~N}_{4}\right)$ filled with and encapsulated by an "inner core" of silicon dioxide $\left(\mathrm{SiO}_{2}\right)$. The structure shown in Figure 2 is formed with only a single photolithography step, as depicted in the process flow shown in Figure 3.

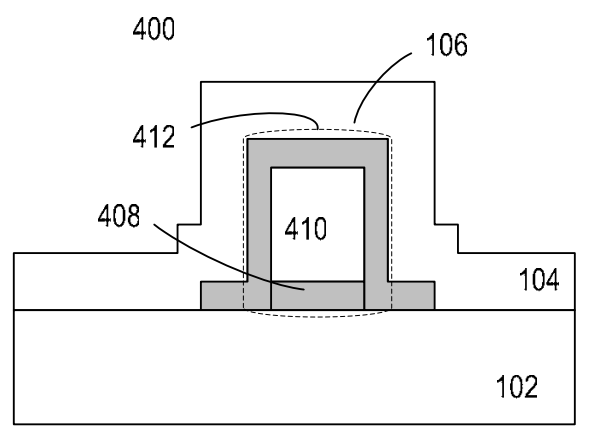

Figure 2: Cross-section of a TriPleX ${ }^{\mathrm{TM}}$ waveguide structure, with $L P C V D \mathrm{Si}_{3} N_{4}$ (in grey) as basic waveguiding layer, filled with and in turn encapsulated by $\mathrm{SiO}_{2}$ (white) [5].
Typically, the nitride outer core has outer dimensions on the order of 1 $\mu \mathrm{m}^{2}$, with its exact characteristics depending strongly upon the desired application. Stoichiometric silicon nitride, deposited using LPCVD, is well-known to have a residual tensile stress of approximately $1 \mathrm{GPa}$. This stress is the root cause of high modal birefringence in classical silicon nitride core high-contrast waveguides. In the structure shown in Figure 2, however, the tensile stress of the nitride is used to compensate the fairly large residual compressive stress of the inner core of LPCVD $\mathrm{SiO}_{2}$ (approximately 3-400 MPa). In this manner, the total stress of the composite layer stack can be controlled and can be made as low or as high as desired. In addition, the thickness of the combined layer stack of nitride and oxide can be considerably larger than the critical layer thickness of $\mathrm{Si}_{3} \mathrm{~N}_{4}$ alone $[5,6]$.

Modal characteristics depend only upon the geometry of the structure, as all composing materials are LPCVD end products with very reproducible characteristics. The whole process is CMOS compatible and extremely cost effective as only one photo lithographical step is required.

Figure 3: Process flow for fabrication of an Ashaped, low modal birefringence TriPleX ${ }^{\mathrm{TM}}$ waveguide: a) Silicon substrate showing LPCVDdeposited stoichiometric layers of silicon nitride and silicon dioxide (deposited using TEOS); b) Ridge waveguide structure as patterned in a single RIE step; c) Waveguide structure comprising an inner core of $\mathrm{SiO}_{2}$ (white) and an outer core of $\mathrm{Si}_{3} \mathrm{~N}_{4}$ (grey). The waveguide structure shown in 2c) further depicts an upper cladding layer of $\mathrm{SiO}_{2}$ that is also deposited using LPCVD; d) Fully formed TriPlex ${ }^{\mathrm{TM}}$ waveguide showing the addition of a plasmadeposited secondary upper cladding (dotted layer).

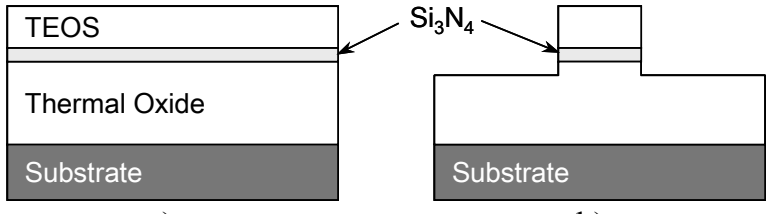

a)

b)

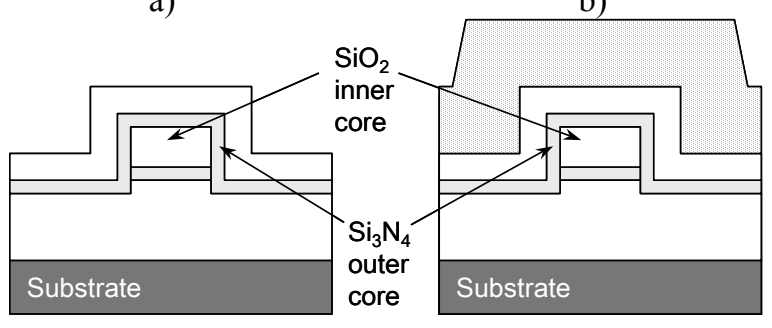

c) d)

Figure 3 depicts the LioniX fabrication procedure for passive optical channels. The process begins with the thermal oxidation of a standard silicon wafer form the lower cladding. This is followed by LPCVD deposition of stoichiometric $\mathrm{Si}_{3} \mathrm{~N}_{4}$ and TEOS $\mathrm{SiO}_{2}$. A single photolithography step is then performed followed by reactive ion etching to form the ridge waveguide seen in Figure 3b. A second layer of LPCVD stoichiometric $\mathrm{Si}_{3} \mathrm{~N}_{4}$ is then deposited followed by a second layer of TEOS $\mathrm{SiO}_{2}$ to form the beginnings of the upper cladding layer. Finally, a thick $\mathrm{SiO}_{2}$ layer is deposited using plasma-enhanced CVD (PECVD) to complete the upper cladding layer. 
By variations in waveguide dimensions, the modal birefringence of a TriPleX ${ }^{\mathrm{TM}}$ waveguide be altered so as to provide a waveguide specific to the desired application. For example, Figure 4a depicts a waveguide suitable for telecom applications wherein modal birefringence is below $10^{-4}$. Figure $4 \mathrm{~b}$ depicts an asymmetrical layout that has been used to form a tunable true time delay (TTD) [7], wherein the channel waveguide is activated by means of heaters fabricated on the top cladding, as will be discussed below.

Figure 4: a) Schematic of a typical telecom waveguide comprising an A-shaped layout with minimal modal birefringence; and b) $a$ non-symmetrical sensing layout with very large modal birefringence so as to strip one polarization mode. The nitride layers are shown in grey.

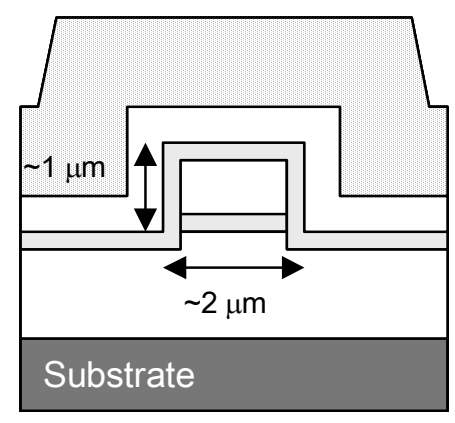

a)

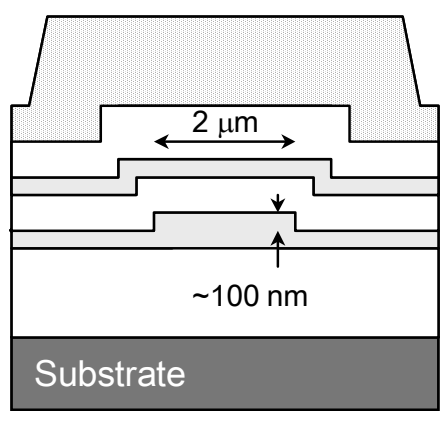

b)

\section{LOW-LOSS WAVEGUIDE PERFORMANCE}

The cross-section of the typical waveguides, as fabricated by LioniX, are shown in Figure 5. Clearly visible is the close agreement with the designs of both structures shown in Figure 4 above.

Figure 5: a) SEM photo of an Ashaped geometry waveguide suitable for telecom applications, with the brighter layers being the silicon nitride. b) SEM photo of a non-symmetrical (sensing layout) waveguide. Here, the darker material is the silicon nitride.

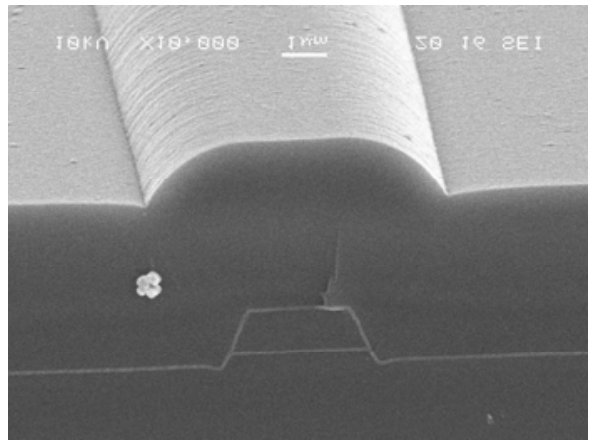

a)

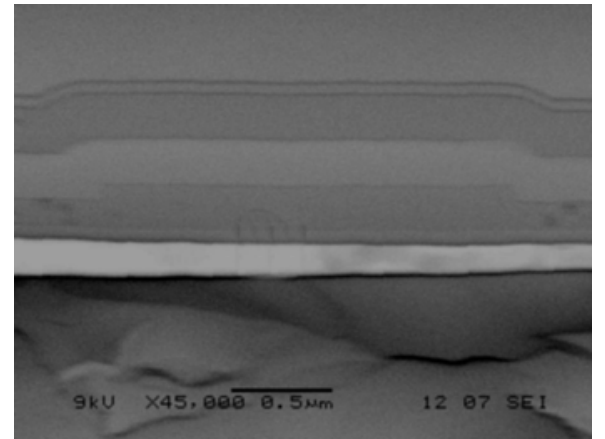

b)

The optical characterization is performed using different techniques, including cut-back and optical low coherence interferometry (OLCI). These measurements are performed on a variety of differently processed wafers, showing a wide range of parameter settings. Key waveguide characteristics are given below in Table 1. 
Table 1. Measured characteristics of two types of single-mode TriPleX $X^{\mathrm{TM}}$ waveguides, A-shaped waveguides for telecom applications as shown in Figures $4 a$ and 5, and non-symmetrical waveguides as shown in Figure $4 b$.

\begin{tabular}{|l|c|c|c|c|}
\hline & $\begin{array}{c}\text { Group } \\
\text { birefringence }\left(\mathrm{B}_{\mathrm{g}}\right)\end{array}$ & $\begin{array}{c}\text { Channel } \\
\text { attenuation } \\
(\mathrm{dB} / \mathrm{cm})\end{array}$ & $\begin{array}{c}\text { Polarization } \\
\text { dependent loss } \\
(\mathrm{PDL}, \text { in } \mathrm{dB})\end{array}$ & $\begin{array}{l}\text { Insertion loss (IL) } \\
\text { without spot size } \\
\text { converter }(\mathrm{dB})\end{array}$ \\
\hline $\begin{array}{l}\text { A-shaped } \\
\text { geometry } \\
\text { (Figure 5a) }\end{array}$ & $4.5 \times 10^{-3}$ & 0.06 & $0.12^{1}$ & $1.4^{2}$ \\
\hline $\begin{array}{l}\text { Non-symm. } \\
\text { sensing layout } \\
\text { (Figure 5b) }\end{array}$ & 0.107 & 0.12 & $0.20^{1}$ & $8.0^{2}$ \\
\hline
\end{tabular}

1: chip length $3 \mathrm{~cm}$

: here, small core fibers were used (MFD of $3.5 \mu \mathrm{m}$ )

: minimal bend radius $\sim 400 \mu \mathrm{m}$

The experimental results provided in Table 1 demonstrate that TriPleX ${ }^{\mathrm{TM}}$ waveguides show very good characteristics for both telecom and sensing applications. Low attenuation is very important for telecom applications, particularly when combined with the very small group birefringence of the channels. Note that this group birefringence is completely adjustable and in good agreement with design expectations for both types of waveguides. . Finally, insertion loss is very good, especially in case of the A-shaped design, wherein the symmetry of the channel modes resulted in nearly circular mode profiles. Fiber-coupling efficiency of $>90 \%$ have been demonstrated with TriPleX ${ }^{\mathrm{TM}}$ waveguides already. For the non-symmetrical sensing layout, insertion loss can be improved significantly by applying properly designed adiabatic taper procedures, resulting in loose-mode segments with very high fiber-to-chip coupling efficiencies.

\section{RESULTS}

\subsection{All-optical True-Time Delay}

Phased-array antennas are commonly used as beam formers and to provide directionality in modern communications systems. Conventional phased array antennas, such as those finding use in wireless telecommunications and navigation, utilize an electronic phase-shifter to control the phase of a signal at each element in the array. Electronic phase-shifters exhibit severe frequency-dependency, however, that makes it difficult to process information over wide frequency bands. $\mathrm{XiO}$ Photonics has developed an all-optical true-time delay (TTD) that promises frequency-independent operation in place of electronic phase-shifters in beam former applications [10]. The fundamental building block of the TTD is an integrated-optic-based ring resonator [8,9]. A ring resonator has a strong group-delay response that enables it to achieve a very stable small time delay, which makes it suitable for providing an adjustable time delay. In particular, a $1 \mathrm{x} 1$ port optical ring resonator acts as an all-pass filter that demonstrates a rapid shift of both phase and group delay near resonance.

Figure 6a depicts a time delay circuit comprising three identical optical ring resonators [7]. The time delay is determined by the tunable optical phase shifter in the ring and the tunable power coupling elements. In order to be suitable for providing phase control in a beam-forming network, a time-shifter must be capable of providing up to 5 nanoseconds of delay, with an accuracy of at least 1 picosecond (ps). Figure $6 \mathrm{~b}$ shows a photo of a single ring-resonator formed in the TriPleX ${ }^{\mathrm{TM}}$ waveguide technology.
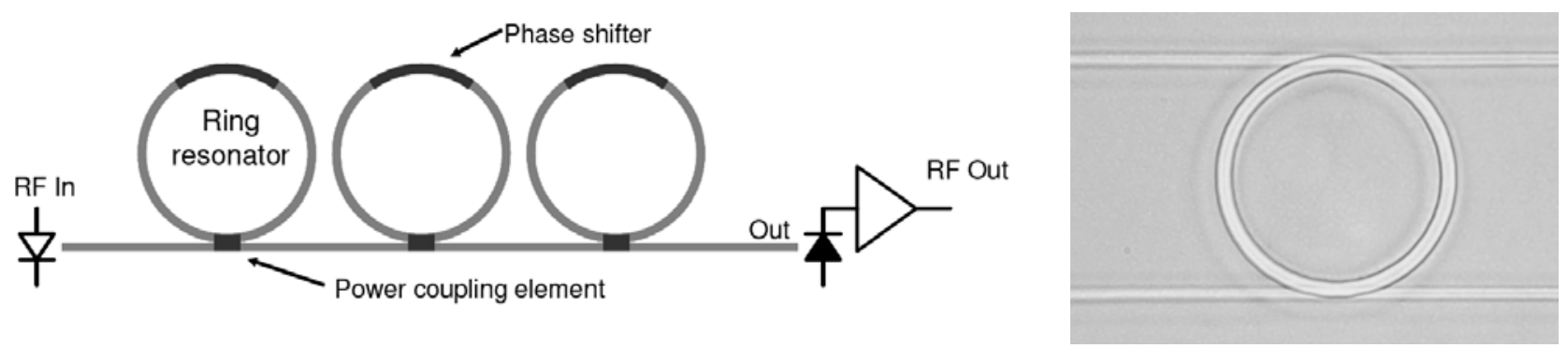
Figure 6: a) A representative time-delay circuit comprising three identically-sized ring resonators. The center ring is a tunable phase-shifter that, when coupled with the remaining two tunable power-coupling elements, provides tunability of up to 5 ns with resolution of 1 ps. b) Photograph of a representative ring resonator.

Figure 7 depicts the phase response and normalized group delay of the time-delay circuit shown in Figure 6a. The normalized group delay is approximately an order of magnitude higher than the roundtrip time of one ring ( $\mathrm{T}_{\text {ring }}$ ). The width of the flat top is approximately one tenth of the Free-Spectral Range (FSR). For example, three rings with a circumference of $2 \mathrm{~mm}$ have a roundtrip time of $0.01 \mathrm{~ns}$ and a FSR of $100 \mathrm{GHz}$, and therefore the group delay is approximately $0.1 \mathrm{~ns}$ over a bandwidth of $10 \mathrm{GHz}$. The intrinsic attenuation of this type of circuit is zero, since there is no optical propagation loss. Figure 8 shows the performance of the three-ring true-time delay circuit. The device was tuned to initiate a delay between 0 and $1.3 \mathrm{~ns}$ with a minimum bandwidth of $500 \mathrm{MHz}$.
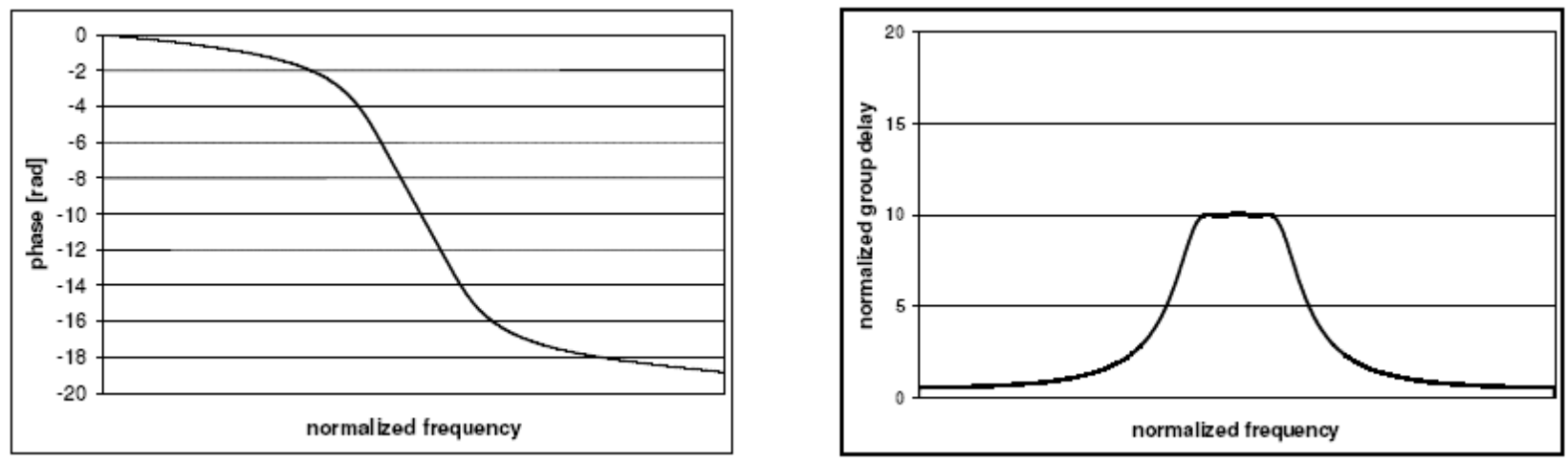

Figure 7: Phase shift and normalized group delay of a three equal-diameter ring resonator circuit. Ring resonators have a periodic frequency response with a period known as the free spectral range (FSR); the frequency response is normalized with respect to this FSR. The group delay is normalized with respect to the roundtrip time of the ring (1/FSR).

Figure 8: Measured delay of a TTD device based on three microring resonators.

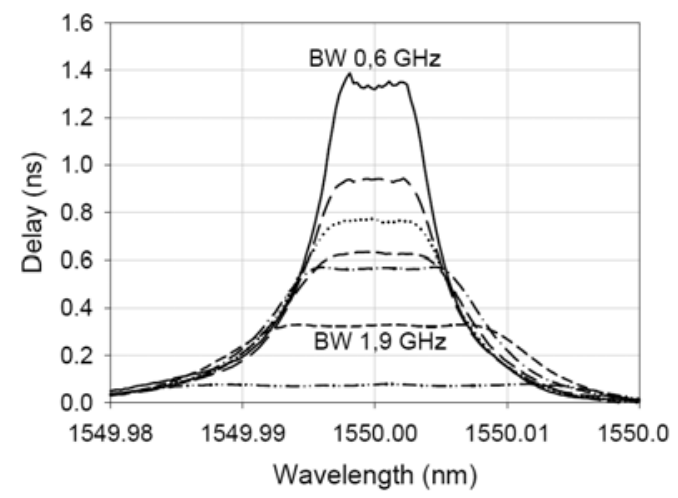

\section{5..2. MZI Optical Sensor Platform}

Figure 9a depicts a schematic drawing of a high-sensitivity optical sensor based on an integrated Mach-Zehnder Interferometer (MZI) based on $\mathrm{Si}_{3} \mathrm{~N}_{4}$ waveguide technology (see also [3]). The MZI sensor comprises two arms, reference arm and sense, each of which carries part of an optical signal injected into the MZI input. The output signal depends upon the relative phase of the light propagating in each arm as the two signals recombine at the output. A small perturbation to the phase of the signal in one arm results in a strong change in output intensity, as is expected for a MZI system. 


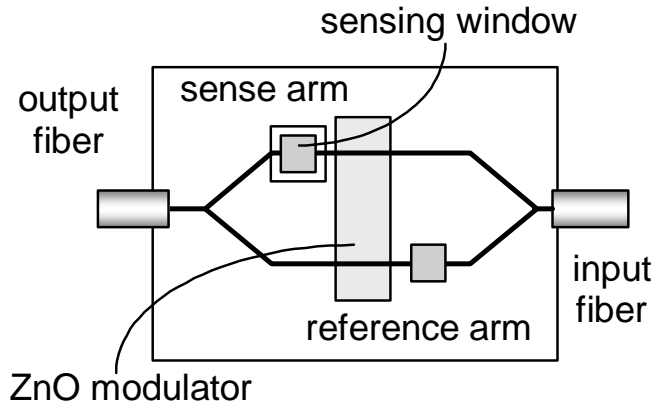

a)

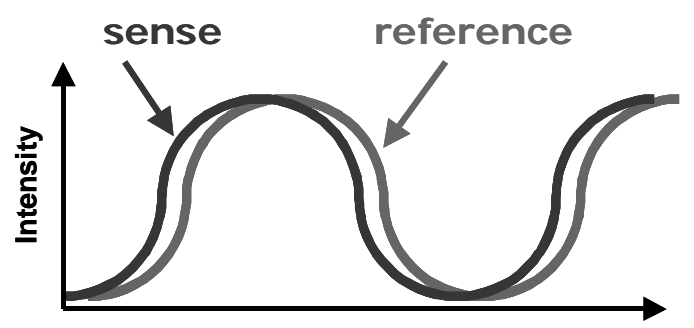

b)

Figure 9: a) Schematic of a Mach-Zehnder Interferometer (MZI) sensor based on a $\mathrm{Si}_{3} \mathrm{~N}_{4}$-core waveguide. Chemo-optical sensitive material, present in the sensing window, changes refractive index in the presence of a target chemical. The refractive index change leads to a phase difference for the optical signals traveling in both arms of the MZI. Modulation of the output signal by the zincoxide electro-optic modulator further enhances the sensitivity of the measurement. b) Representation of the optical signal traveling in the sense and reference arms.

In order to form the basis of a chemical sensor, each arm of the MZI contains a substantially identical window, onto which a layer of chemically-sensitive or bioactive material is deposited. The material is chosen so as to be sensitive to only the presence of one target chemical. The material on the sensor arm is open to the ambient environment, while the material on the reference arm is protected from exposure. In the presence of the target chemical, the refractive index of the chemically sensitive material undergoes a change, thereby changing the phase of the optical signal in the sense arm. A wide array of selectively-sensitive materials are available, and thus sensors for any one of a wide variety of gasses, liquids, compounds, or organic materials can be fabricated.

Since high-contrast waveguides enable high-density integration of multiple integrated optic circuits, multiple sensors can be fabricated on a single chip. Figure 10a shows a photo of a high-contrast waveguide chip that comprises five individual MZI sensor circuits, of which (in this case) only one is selected. The sensor chip is temperature insensitive, low power, and also immune to electro-magnetic interference. Figure 10b shows the corresponding entire sensing system, as currently being commercialized by Optisense BV.

Figure 10: a) Photo of a commercially-available, multiple MZI carrying optical sensor based on LioniX's MZI technology [11]; and, b) the total system including electronics and laptop for on-line readout.

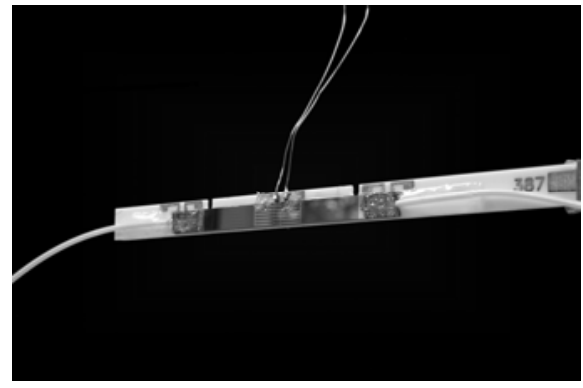

a)

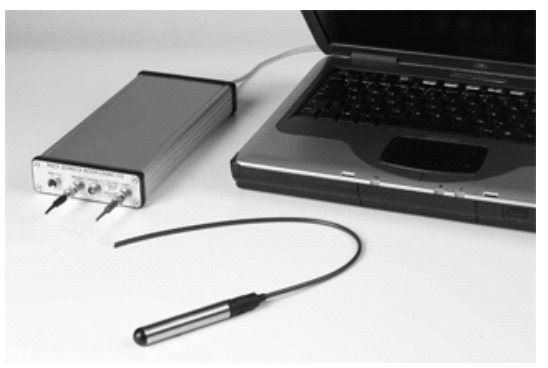

b)

In the LioniX optical chip shown in Figure 10a, the silicon nitride core is employed so as to strip one polarization mode from the optical signal and thus enhance SNR. The thin nature of the cladding and easily accessible propagating mode of the high-contrast waveguide enables high-sensitivity at low cost. Here, the use of TriPleX ${ }^{\mathrm{TM}}$ technology will further improve the characteristics of this optical chip.

As discussed, this MZI sensor system can be designed to sense any of a variety of chemicals and/or compounds including salmonella, SARS virus, legionella, hormones, pollutants, etc.. 


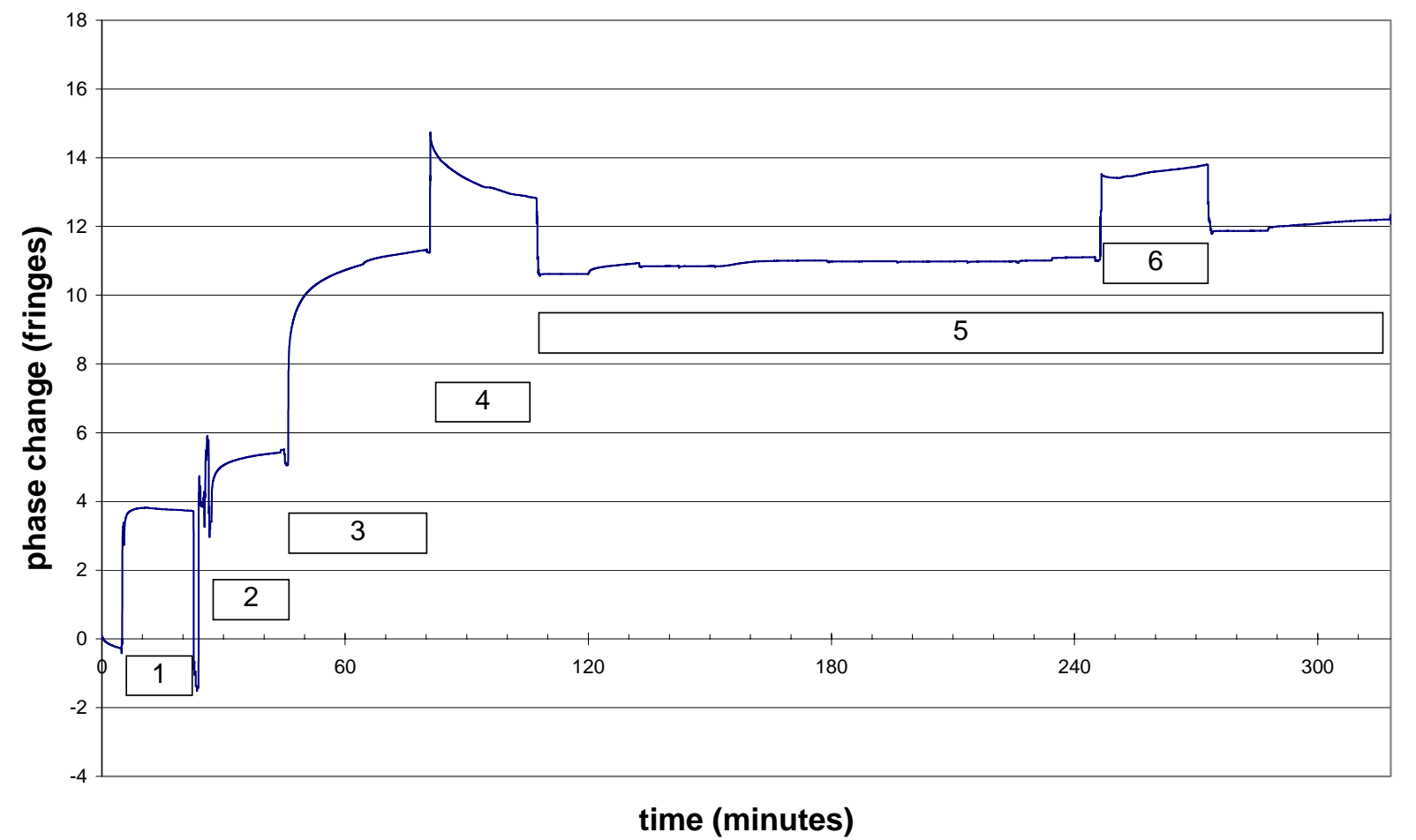

Figure 11: MZI output signal showing an antibody coating procedure resulting in antigen sensitivity. In this case, the afla-toxine afla-HRP is detected.

Figure 11 shows a typical output signal of an OptiSense sensor, which is treated so as to be sensitive to afla-HRP (an aflotoxine), Here, the sensor output is given in fringes, a fringe being equal to a phase chance of $2 \pi$. In step 1 , a cleaning procedure is performed, (using piranha, ethanol and demi water), followed by a silane coating. In step 2 the sensor surface is activated using the glutaric dialdehyde procedure, after which in step 3 the antibody $\alpha$-afla-HRP (10 $\mu \mathrm{g} / \mathrm{ml}$ ) is added: clearly visible is typical curve of the binding process. After that, a blocking procedure is performed, followed by several antigen incubations at increasing concentrations (step 5). During this repeated incubation, a regeneration step (ethylene glycol in demi water) is performed to check the antibody activity (step 6).

\section{CONCLUSIONS}

New high-contrast surface waveguide technologies show significant promise for application in many areas, including telecom and optical sensing. These new waveguide technologies do not suffer from the high cost of fabrication and packaging that have limited the deployment of conventional $\mathrm{SiO}_{2}$-based waveguide systems. In addition, they also exhibit performance that approaches and, in some cases, equals that of conventional waveguides.

Optical sensors, based on silicon nitride and/or silicon oxynitride waveguide cores, have been developed and are being readied for commercial deployment. The development of a new composite waveguide technology, the TriPleX ${ }^{\mathrm{TM}}$ waveguide, provides a path for improved optical sensor performance as well as low-cost, low-polarization-sensitivity telecommunications devices. Early experiments on typical geometries show very promising waveguide characteristics (attenuation $« 0.5 \mathrm{~dB} / \mathrm{cm}, \mathrm{IL} \leq-2 \mathrm{~dB}, \mathrm{PDL} \ll 1 \mathrm{~dB}$, bend radius $« 1 \mathrm{~mm}$ ).

\section{REFERENCES}

[1] J.C. Chon, B. Jian, and J. Bautista, "High-capacity, high-speed DWDM and NWDM Optical Devices for Telecom and Datacom Applications," Proc. Photonics West 2001, San Jose, CA, 2001.

[2] MIT Microphotonics Center, “MIT Photonics Technology Roadmap 2005 - Silicon Optoelectronics”, pp. 23-24, (2005). 
[3] R.G. Heideman, P.V. Lambeck, "Remote chemo-optical sensing with extreme sensitivity: design, fabrication and performance of a pigtailed integrated optical phase-modulated Mach-Zehnder interferometer", Sensors and Actuators B, vol.61, p.100-127, 1999.

[4] R.G. Heideman, A. Melloni, M. Hoekman, A. Borreman, A. Leinse, and F. Morichetti, "Low-loss, high-contrast optical waveguides based on CMOS-compatible LPCVD processing: Technology and Experimental Results," Proc. Leos Benelux Ch., 2005.

[5] U.S. Patent Application 10/756627-001, "Low modal birefringent waveguides and methods of fabrication,"

[6] M. Melchiorri, si,N.Daldosso, F.Sbrana and L.Pave, "Propagation losses of silicon nitride waveguides in the near-infrared range," Applied Physics Letters, Vol. 86, pp. 121111-13 (2005).

[7] C.G.H.Roeloffzen, L.Zhuang, R.G.Heideman, A.Borreman, and W. van Etten, "Ring resonator-based tunable optical delay line in LPCVD waveguide technology", Proc. Leos Benelux Ch., 2005.

[8] A.Driessen, D.H.Geuzebroek, H.J.W.M.Hoekstra, H.Kelderman, E.J.Klein, D.J.W.Klunder, C.G.H. Roeloffzen, F.S.Tan, E.Krioukov, C.Otto, H.Gersen, N.F.van Hulst and L.Kuipers, "Microresonators as building blocks for VLSI photonics", AIP conf. Proc., Vol. 709, pp.1-8, 2003.

[9] D.H.Geuzebroek, E.J.Klein, H.Kelderman and A.Driessen, "Wavelength tuning and switching of a thermo-optic microring resonator", proc. ECIO 2003, pp. 395-398, 2003.

[10] www.xiophotonics.com

[11] www.lionixbv.nl/download/pdf/Leaflet\%20MZI\%20versie3.pdf 